\title{
Cryo-EM Structure of Nipah Virus Fusion Glycoprotein in Complex with a Monoclonal Antibody Reveals Mechanism of Neutralization
}

\author{
Ha V. Dang ${ }^{1}$, Yee-Peng Chan ${ }^{2 *}$, Young-Jun Park ${ }^{1}$ and Christopher C. Broder ${ }^{2}$ and David Veesler ${ }^{*}$ \\ 1. Department of Biochemistry, University of Washington, Seattle, Washington, USA. \\ 2. Department of Microbiology and Immunology, Uniformed Services University, Bethesda, Maryland, \\ USA. \\ * Corresponding author: dveesler@uw.edu
}

Nipah virus $(\mathrm{NiV})$ and Hendra virus $(\mathrm{HeV})$ are zoonotic paramyxoviruses with a high fatality rate in humans due to devastating neurological and respiratory complications [1]. Despite the fact that NiV and $\mathrm{HeV}$ have been causing recurring outbreaks in South/Southeast Asia and Australia since their first emergence in the 1990s, there are currently no approved vaccines or treatments for human against these dangerous viruses. WHO placed NiV and $\mathrm{HeV}$ on the 2018 list of priority disease-causing pathogens along with other dangerous viruses such as Lassa virus and Ebola virus, emphasizing the urgent demand for therapeutics to counteract $\mathrm{NiV}$ and $\mathrm{HeV}$ before a potential future outbreak.

NiV belongs to the Paramyxovirus family, which includes a number of disease-causing pathogens in human such as measles, mumps, pneumoviruses and parainfluenza viruses. Paramyxoviruses are enveloped viruses. Their entry into host cells requires fusion of the viral and host membranes, which is mediated by the interplay between two glycoproteins on its surface: a tetrameric attachment protein that recognizes specific receptors on the host cell and a trimeric fusion protein that performs fusion of host and viral membranes. These two glycoproteins of $\mathrm{NiV}$ are known as $\mathrm{G}$ and $\mathrm{F}$, respectively. NiV G and $\mathrm{F}$ glycoproteins are the main antigens presented to the hosts during infection and are therefore attractive targets for antiviral strategies and vaccine designs.

A monoclonal antibody (mAb), known as 5B3, isolated from a mouse immunized with a soluble construct of $\mathrm{NiV} \mathrm{F}$, is specific for the prefusion conformation of $\mathrm{NiV} F$ and neutralizes against both $\mathrm{NiV}$ and $\mathrm{HeV}$ in a pseudotyped virus neutralization assay [2]. In order to understand how 5B3 is able to block the function of one of $\mathrm{NiV}$ essential glycoproteins, we aimed to solve the structure of $\mathrm{NiV} \mathrm{F}$ in complex with 5B3 using cryo-EM. We expressed and purified a soluble ectodomain construct of NiV F stabilized in the prefusion conformation from mammalian cells and formed a complex with the Fab domain of 5B3. The complex displayed severe preferred orientation for the top view along its C3 symmetry axis when vitrified with the conventional method on holey C-flat grids (Figure 1A), which hindered the determination of a 3D-reconstruction to high resolution. To overcome the preferred orientation problem, we used holey C-flat grids coated with a layer of ultra-thin carbon. The complex adopts multiple different orientations on thin-carbon grids as seen from 2D class averages (Figure 1B). $3 \mathrm{D}$ reconstruction of this dataset resulted in an isotropic map at $3.5 \AA$ (Figure $2 \mathrm{~A}-\mathrm{B}$ ). We also crystallized and solved the structure of the Fab domain of 5B3 1.9 $\AA$. To build an atomic model of the complex, we used this crystal structure of $5 \mathrm{~B} 3 \mathrm{Fab}$ and the published crystal structure of NiV F [3] for initial fitting into our $3.5 \AA$ cryo-EM map and further refined the model in Rosetta (Figure $2 \mathrm{C}$ ).

Our cryo-EM structure is the first to reveal the near-atomic details of the binding mode between NiV F glycoprotein and a neutralizing antibody and suggests that 5B3 neutralizes against NiV by locking the F protein in its prefusion conformation by covering an extensive quaternary epitope on NiV F (Figure 2D). 
Comparing with the post-fusion structure of the fusion glycoprotein from PIV5, a closely related virus, we found that segments comprising the epitope of $5 \mathrm{~B} 3$ on $\mathrm{NiV} F$ are in close proximity in NiV F prefusion structure but become far apart in the post-fusion conformation. 5B3 therefore may act as a molecular staple to stabilize the prefusion conformation of NiV F and thereby block its membrane fusion activity. This mechanism of action is supported by a refolding experiment where NiV F refolding is triggered by tryptic cleavage and thermal incubation at $50^{\circ} \mathrm{C}$ but fails in the presence of m5B3. NiV F refolding can be partially rescued by raising the incubating temperature, indicating that $\mathrm{m} 5 \mathrm{~B} 3$ provides stabilization to the prefusion conformation (data not shown). The atomic model of our structure explains how $5 \mathrm{~B} 3$ is specific to the prefusion conformation of $\mathrm{NiV} \mathrm{F}$ and provides strategies to develop 5B3 into a therapeutic treatment against both $\mathrm{NiV}$ and $\mathrm{HeV}$.

References:

[1] World Health Organization, http://www.who.int/csr/disease/nipah/en/ (Accessed on Feb 14, 2019).

[2] YP Chan et al., Journal of Virology 86(21) (2012), p. 11457.

[3] K Xu et al., PLoS Pathogens 11(12) (2015), e1005322.
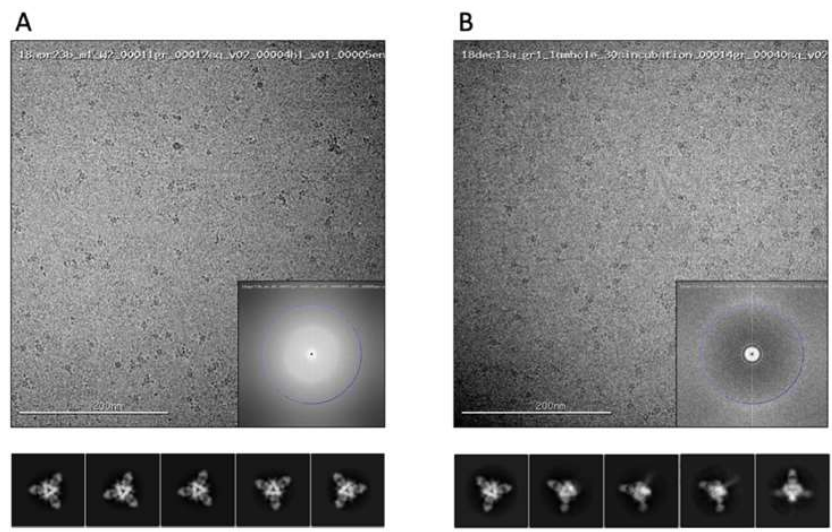

Figure 1. Examples of a micrograph with the corresponding power spectrum (right corner) and $2 \mathrm{D}$ class averages (below) of from datasets of $\mathrm{NiV} \mathrm{F}$ and 5B3 complex when vitrified using Vitrobot on (A) holey C-flat grids and (B) holey C-flat grids coated with a layer of ultra-thin carbon.

A

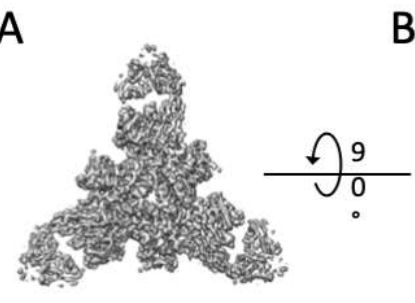

B

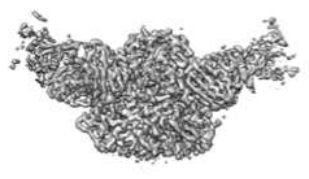

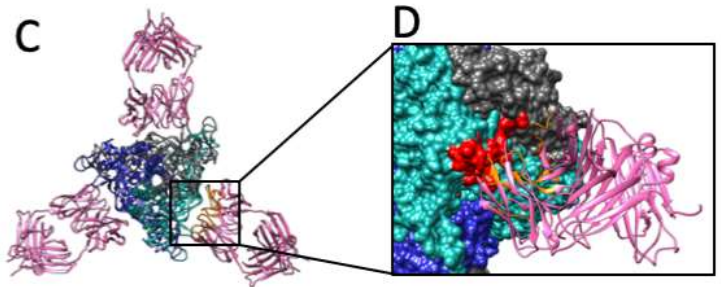

Figure 2. 3D map at $3.5 \AA$ of $\mathrm{NiV} \mathrm{F}$ and mAb 5B3 complex from the (A) top view (looking towards the viral membrane) and (B) side view. (C) Ribbon diagrams showing the atomic model of the complex as oriented in (A). (D) Epitope of $5 \mathrm{~B} 3$ on NiV F is quateAnary (i.e: involves two adjacent protomers on $\mathrm{NiV} \mathrm{F}$ ). NiV F is presented as surface filtered to $4 \AA, \mathrm{mAb} 5 \mathrm{~B} 3$ is presented in ribbon diagram. Grey, green and blue: F protomers; pink: m5B3 Fab domain with CDR loops highlighted in red; yellow: surface area of NiV F buried by m5B3. 